\title{
A different perspective on alternative cleavage and polyadenylation
}

\section{Chuan Xu and Jianzhi Zhang (D)}

In a Review in this journal (Alternative cleavage and polyadenylation in health and disease. Nat. Rev. Genet. 20, 599-614 (2019))' ${ }^{1}$, Gruber and Zavolan summarized recent research on alternative polyadenylation (APA). Under the presumption that APA is beneficial, the authors presented cases where APA produces mRNAs of different functions or regulations from the same gene and discussed the APA defects that can cause disease. However, genome-scale evidence suggests that APA arises largely from molecular error and is generally nonadaptive $^{2}$, which runs counter to the notion that APA has a positive functional impact.

Support for the notion that APA enhances useful function or regulation is available only anecdotally ${ }^{1,3-5}$. Genomic studies have failed to detect a clear relationship between APA and mRNA stability and translatability ${ }^{6}$ or protein concentration ${ }^{7}$. These and other observations prompted the proposal that most genes have only one optimal polyadenylation site, which is typically the most frequently used site (hereafter referred to as the major site), and that APA occurs largely owing to polyadenylation error ${ }^{2}$. This error hypothesis predicts a number of global patterns of APA that have been verified in multiple tissues from five mammals ${ }^{2}$. Below, we estimate the fraction of deleterious APA that occurs in humans by an established method ${ }^{8-10}$.

Given a polyadenylation error rate, more molecules of non-optimal mRNA are produced from more highly expressed genes. This difference creates stronger selection against polyadenylation error in more highly expressed genes, causing the extent of APA to decrease with gene expression level ${ }^{2}$. Under the assumption that polyadenylation error has not been selectively removed at all in the least expressed genes but has been completely removed in the most expressed genes, a conservative estimate of the fraction of deleterious polyadenylation can be obtained by 1 minus the extent of APA that occurs in the 20 most expressed genes relative to that in the 20 least expressed genes. This fraction turns out to be $\sim 93 \%$ (based on figure $1 \mathrm{~A}$ in REF. ${ }^{2}$ ), potentially explaining why beneficial APA is not observed at the genomic scale. Note that a genome-wide difference in the length of $3^{\prime}$ untranslated regions among tissues or developmental stages is not evidence for adaptation because such a difference could result from expression changes of a small number of trans-factors ${ }^{1}$.

The mechanisms by which alterations of APA have an impact on health and disease differ under the adaptive and error hypotheses. A disruption of polyadenylation at the major site is expected to be harmful under both hypotheses. By contrast, increasing polyadenylation at an existing non-major site or creating a new polyadenylation site is not expected to be pathogenic under the adaptive hypothesis, but will probably be so under the error hypothesis. Thus, the finding that gaining a proximal polyadenylation site in IRF5 causes systemic lupus erythematosus ${ }^{11}$ is more readily explained by the error hypothesis than the adaptive hypothesis.

In summary, the error hypothesis provides a different perspective on the origin, biological significance and disease relevance of APA than the adaptive view held by Gruber and Zavolan ${ }^{1}$. Considering both perspectives will likely be more productive in APA research than holding only one view, especially when this view fails the test of genomics.

There is a reply to this letter by Gruber, A. J. \& Zavolan, M. Nat. Rev. Genet. https://doi.org/ 10.1038/s41576-019-0199-y (2019).
Chuan Xu and Jianzhi Zhang (iD)* Department of Ecology and Evolutionary Biology. University of Michigan, Ann Arbor Michigan, USA

*e-mail: jianzhi@umich.edu https://oi.org/10.1038/s41576-019-0198-z

1. Gruber, A. J. \& Zavolan, M. Alternative cleavage and polyadenylation in health and disease. Nat. Rev. Genet 20, 599-614 (2019).

Xu, C. \& Zhang J. Alternative polyadenylation of mammalian transcripts is generally deleterious, not adaptive. Cell Syst. 6, 734-742 (2018).

3. Di Giammartino, D. C., Nishida, K. \& Manley, J. L. Mechanisms and consequences of alternative polyadenylation. Mol. Cell 43, 853-866 (2011).

4. Mayr, C. Evolution and biological roles of alternative 3'UTRs. Trends Cell Biol. 26, 227-237 (2016).

5. Tian, B. \& Manley, J. L. Alternative polyadenylation of mRNA precursors. Nat. Rev. Mol. Cell Biol. 18, 18-30 (2017).

6. Spies, N., Burge, C. B. \& Bartel, D. P. 3' UTR-isoform choice has limited influence on the stability and translational efficiency of most mRNAs in mouse fibroblasts. Genome Res. 23, 2078-2090 (2013).

7. Gruber, A. R. et al. Global 3' UTR shortening has a limited effect on protein abundance in proliferating T cells. Nat. Commun. 5, 5465 (2014).

8. Li, C. \& Zhang, J. Stop-codon read-through arises largely from molecular errors and is generally nonadaptive. PLOS Genet. 15, e1008141 (2019).

9. Xu, C., Park, J. K. \& Zhang, J. Evidence that alternative transcriptional initiation is largely nonadaptive. PLOS Biol. 17, e3000197 (2019).

10. Saudemont, B. et al. The fitness cost of mis-splicing is the main determinant of alternative splicing patterns. Genome Biol. 18, 208 (2017).

11. Graham, R. R. et al. Three functional variants of IFN regulatory factor 5 (IRF5) define risk and protective haplotypes for human lupus. Proc. Natl Acad. Sci. USA 104, 6758-6763 (2007).

\section{Author contributions}

C.X. researched the literature. C.X. and J.Z. provided substantial contributions to discussions of the content, wrote the article, and reviewed and/or edited the manuscript before submission.

\section{Competing interests}

The authors declare no competing interests.

\section{Reply to 'A different perspective on alternative cleavage and polyadenylation'}

\section{Andreas J. Gruber(i) and Mihaela Zavolan (1)}

In their Correspondence (A different perspective on alternative cleavage and polyadenylation. Nat. Rev. Genet. https://doi.org/ 10.1038/s41576-019-0198-Z (2019)) $)^{1}$ Xu and Zhang state that we have written our recent Review on alternative cleavage and polyadenylation (APA) in health and disease (Alternative cleavage and polyadenylation in health and disease. Nat. Rev. Genet. 20, 599-614 (2019))2 under the presumption that APA is generally beneficial or adaptive. This is not the case.

As we and others have found in early genomics studies of alternative splicing, many alternative isoforms can be explained by 'noise' in the processing of closely spaced sites, whose relative efficiency of processing could be predicted from the sequence ${ }^{3}$. More broadly, it is no longer disputed that stochasticity is pervasive at all steps of gene expres$\operatorname{sion}^{4}$ and that deep sequencing has brought to light a large number of rare variants with little functional relevance. The extensive discussion around the results of the ENCODE project ${ }^{5}$ illustrates this point (for example, REF. ${ }^{6}$ ). Thus, in contrast with what $\mathrm{Xu}$ and Zhang suggest, we are mindful that many of the unique APA variants observed in a genome-wide study are likely the result of imprecision in the selection of processing sites. For this reason, in our studies, we cluster closely spaced RNA $3^{\prime}$ ends 
that very likely result from imprecise processing, and we typically also require that analysed sites be identified in multiple experiments.

Beyond this, it is important to stress that in the absence of a specific model, such as the one we had for splicing, it is unclear what sort of patterns of isoform expression to expect. Furthermore, 'noisy' expression may be, in fact, beneficial in some situations ${ }^{7,8}$. Establishing whether particular molecular events are adaptive is notoriously difficult, and we thus avoided making claims to that effect in our Review. Instead, we focused on patterns that, in our opinion, warrant further investigation. Specifically, we highlighted (1) the coherent shift toward usage of proximal poly(A) sites in cancers and its potential mechanisms and (2) some examples of changes in poly(A) site usage for which the relationship to the disease has strong experimental support. How a systematic shift towards proximal poly(A) site usage arises in cancers is unclear. We and others hypothesized that specific regulators are involved (for example, REFS ${ }^{9-12}$ ), but the question is by no means answered. Once generated, either owing to a general defect in polyadenylation or to the action of specific regulatory proteins, APA isoforms can be co-opted in pathogenic processes, as the IRF5 example illustrates ${ }^{13}$. Identifying such cases, and especially predicting at what level they affect cellular function, is not trivial. Our efforts to approach this issue are described in a previous study ${ }^{14}$.

To conclude, we do not hold the view that the majority of unique APA isoforms that are observed in a deep-sequencing study have specific functions. While our Review highlights some known examples of functional isoforms, more remain to be uncovered, as even those APA isoforms that result from noisy processing can play roles in pathogenic processes. Further progress in the field requires approaches for identifying functional variants and the mechanisms that generate them.
Andreas J. Gruber (iD) ${ }^{1 *}$ and Mihaela Zavolan (iD ${ }^{2 *}$ 'Oxford Big Data Institute, Nuffield Department of Medicine, University of Oxford, Oxford, UK. ${ }^{2}$ Computational and Systems Biology, Biozentrum, University of Basel, Basel, Switzerland. *e-mail: andreas.gruber@bdi.ox.ac.uk; mihaelazavolan@unibas.ch https://doi.org/10.1038/s41576-019-0199-y

1. Xu, C. \& Zhang, J. A different perspective on alternative cleavage and polyadenylation. Nat. Rev. Genet. https:// doi.org/10.1038/s41576-019-0198-z (2019).

2. Gruber, A. J. \& Zavolan, M. Alternative cleavage and polyadenylation in health and disease. Nat. Rev. Genet. 20, 599-614 (2019).
3. Chern, T.-M. et al. A simple physical model predicts small exon length variations. PLOS Genet. 2, e45 (2006)

4. Raser, J. M. \& O'Shea, E. K. Noise in gene expression: origins, consequences, and control. Science 309, 2010-2013 (2005).

5. ENCODE Project Consortium. An integrated encyclopedia of DNA elements in the human genome. Nature 489, 57-74 (2012).

6. Eddy, S. R. The C-value paradox, junk DNA and ENCODE. Curr. Biol. 22, R898-899 (2012).

7. Eldar, A. \& Elowitz, M. B. Functional roles for noise in genetic circuits. Nature 467, 167-173 (2010)

8. Wolf, L., Silander, O. K. \& van Nimwegen, E. Expression noise facilitates the evolution of gene regulation. elife 4, e05856 (2015).

9. Elkon, R. et al. E2F mediates enhanced alternative polyadenylation in proliferation. Genome Biol. 13 , R59 (2012).

10. Jenal, M. et al. The poly(A)-binding protein nuclear 1 suppresses alternative cleavage and polyadenylation sites. Cell 149, 538-553 (2012).

11. Hwang, H.-W. et al. PAPERCLIP identifies microRNA targets and a role of CstF64/64tau in promoting non-canonical poly(A) site usage. Cell Rep. 15, 423-435 (2016).

12. Gruber, A. J. et al. A comprehensive analysis of $3^{\prime}$ end sequencing data sets reveals novel polyadenylation signals and the repressive role of heterogeneous ribonucleoprotein C on cleavage and polyadenylation. Genome Res. 26, 1145-1159 (2016).

13. Graham, R. R. et al. Three functional variants of IFN regulatory factor 5 (IRF5) define risk and protective haplotypes for human lupus. Proc. Natl Acad. Sci. USA 104, 6758-6763 (2007)

14. Gruber, A. R. et al. Global 3' UTR shortening has a limited effect on protein abundance in proliferating T cells. Nat. Commun. 5, 5465 (2014).

Author contributions

The authors contributed equally to all aspects of the article.

Competing interests

The authors declare no competing interests. 